\title{
PESO-COMPRIMENTO E FATOR DE CONDIÇÃO RELATIVO DE BRYCONAMERICUS IHERINGII EM RIACHOS DO PARANAPANEMA
}

\author{
Dyego Leonardo Ferraz Caetano* \\ Daniela Ribas Jané**
}

RESUMO: Foram analisados a relação peso-comprimento e o Fator de Condição Relativo (Kn) de peixes da espécie Bryconamericus iberingii capturados em três riachos da bacia do rio das Cinzas, Alto rio Paraná, para estimar, respectivamente, o tipo de crescimento e o bem-estar das populações. Análises de Variância (ANOVA Unifatorial) foram utilizadas para verificar diferenças significativas nos valores de peso, comprimento e Kn entre os riachos, e análises de correlação (Spearman) indicaram a relação entre esses parâmetros supracitados e os fatores físicos e químicos e densidade populacional nos riachos. Os indivíduos do riacho Ubá, considerado o mais preservado com relação à vegetação ripária entre os três estudados, apresentaram maiores valores de peso, comprimento e Kn. Os indivíduos dos riachos Água dos Anjos e Monjolinho exibiram crescimento alométrico negativo, com maior acréscimo em comprimento (mm), e os do riacho Ubá, alométrico positivo, com ganho maior em peso $(\mathrm{g})$. $\mathrm{O}$ Kn se correlacionou $(p<0,05)$ negativamente com a densidade e a condutividade, e a condutividade baixa, por consequência, teve correlação com a alta extensão da mata ripária. Portanto, o presente trabalho mostra indícios de que os parâmetros de relação peso-comprimento e de Kn de peixes de riacho podem ser influenciados significativamente pelas características ambientais relacionadas principalmente à vegetação marginal, podendo ser boas métricas complementares para entender a resposta desses organismos às alterações antrópicas.

PALAVRAS-CHAVE: Densidade e peso de peixes; Ecologia de riacho; Fatores abióticos de riachos; Vegetação ripária.

Biólogo. Doutor em Biologia das Interações Orgânicas pela Universidade Estadual de Maringá (UEM). Docente colaborador do colegiado de Ciências Biológicas da Universidade Estadual do Norte do Paraná (UENP), Brasil, e pesquisador do Grupo de Estudos e Pesquisa em Recursos Hídricos e Ecologia Aplicada (GEPRHEA/ UENP), Brasil.

** Discente do curso de Medicina Veterinária das Faculdades Integradas de Ourinhos (FIO, Brasil. 


\section{WEIGHT-LENGTH AND RELATIVE CONDITION FACTOR OF Bryconamericus iheringii IN STREAMS OF THE PARANAPANEMA RIVER, BRAZIL}

ABSTRACT: Relationship weight-length and the Relative Condition Factor (Kn) of fish species Bryconamericus iberingii were analyzed. Fish were captured in three streams of the Cinzas River basin, high river Paraná, to determine growth rate and well-being of populations. Analyses of Variance (Unifactorial ANOVA) verified significant differences in weight, length and Kn between streams, whereas Spearman correlation analyses indicated the relationship between the above mentioned parameters and physical and chemical factors and population density in the streams. Specimens from the stream Ubá, the most preserved stream among the three analyzed with regard to riparian vegetation, had greater weight, length and Kn. Specimens from streams Água dos Anjos and Monjolinho showed negative allometric growth, with greater increase in length $(\mathrm{mm})$, whereas specimens from the stream Ubá had positive allometric growth, with greater increase in weight (g). Kn was co-related $(p<0.05)$ negatively with density and conductivity. Low conductivity was co-related with high extension of riparian vegetation. Current analysis reveals that parameters for weight-length and $\mathrm{Kn}$ in stream fish specimens may be affected significantly by environmental characteristics, mainly related to bank vegetation. They may be good complementary measures for responses of organisms to anthropic changes.

KEY WORDS: Fish density and weight; Stream ecology; Biotic factors of streams; Riparian vegetation.

\section{INTRODUÇÃO}

Estudos que consideram o Fator de Condição Relativo (KN; LE CREN, 1951) e o quociente peso-comprimento de peixes podem gerar informações indiretas sobre a maturidade, reprodução, nutrição e saúde das populações (GUIDELLI et al., 2011; CIFUENTES et al., 2012). O Kn é um índice ecológico que indica o bemestar do peixe por meio do quociente entre o peso observado e o esperado para determinado comprimento (ANDRADE-TALMELLI; FENERICH-VERANI; VERANI, 1999), e a relação peso-comprimento gera resultados importantes referentes ao tipo de crescimento das populações estudadas (SCHNEIDER; LAARMAN; 
GOWING, 2000; RÊGO et al., 2008). Esses parâmetros de peso-comprimento são utilizados com mais frequência em populações de peixes de importância econômica (AGBOOLA; ANETEKHAI, 2008; TREER et al., 2008), e justamente por isso, riachos são proporcionalmente menos estudados do que rios, lagos e represas, pois não apresentam espécies economicamente importantes (UIEDA; CASTRO, 1999). No entanto, os riachos possuem importância fundamental para as bacias hidrográficas às quais pertencem, principalmente com relação à hidrologia e à disponibilidade de recursos para as comunidades bióticas (VANNOTE et al., 1980; ALEKSEEVSKII et al., 2003; RÊGO et al., 2008).

As assembleias de peixes de riacho têm sua estrutura trófica e dinâmica influenciadas pelas variáveis físicas, químicas e hidrológicas dos babitat aquáticos (CASATTI, 2014). Algumas dessas variáveis abióticas que influenciam a ecologia de espécies de peixes de riacho são: largura, profundidade, velocidade da correnteza, tipo de substrato, potencial hidrogeniônico $(\mathrm{pH})$, temperatura, turbidez, condutividade e oxigênio dissolvido (SÚAREZ et al., 2011; GONÇALVES; BRAGA, 2012; ZENI; ROSA et al., 2016).

No entanto, a biota dos ecossistemas aquáticos também é influenciada por fatores externos (das margens), dentre os quais se destaca a extensão da vegetação ripária. Áreas florestais apresentam importantes funções para a integridade de sistemas aquáticos e para a ecologia dos peixes. Quaisquer alterações relacionadas à diminuição da vegetação ripária, seja em áreas de preservação permanente (APP), em reservas legais ou em áreas de altitude elevada, podem levar à diminuição na riqueza de espécies, no aumento da dominância e da homogeneização da fauna e na diminuição da biomassa dos peixes (CASATTI, 2010; RIBEIRO; TERESA; CASATTI, 2016).

Bryconamericus iberingii (Boulenger, 1887) é uma espécie de peixe pertencente à família Characidae bastante comum em riachos do sistema do Alto rio Paraná (SHIBATTA et al., 2002; ORICOLLI; BENNEMANN, 2006; ROSA et al., 2016; CAETANO; OLIVEIRA; ZAWADZKI, 2016), sendo que sua distribuição geográfica abrange, além da bacia do rio Paraná, as bacias da Laguna dos Patos e do rio Uruguai (GRAÇA; PAVANELLI, 2007). Essa espécie, também conhecida como lambarizinho ou pequira, possui pequeno porte (comprimento total máximo de $60 \mathrm{~mm}$ ) e apresenta 
hábito alimentar generalista (ORICOLLI; BENNEMANN, 2006) com tendência à herbivoria (HAHN; FUGI; ADRIAN, 2004).

Sendo assim, torna-se importante entender como as espécies de peixes respondem (em sua biologia e ecologia) às alterações físicas, químicas e hidrológicas em riachos, e também às modificações em suas margens (relacionadas principalmente à mata ripária). Dentro deste contexto, o objetivo do presente trabalho é analisar e comparar a relação peso-comprimento e o Fator de Condição Relativo (Kn) de peixes da espécie Bryconamericus iberingii presentes em riachos da bacia do rio das Cinzas, Alto rio Paraná, verificando a correlação do peso e do Kn dos indivíduos com a densidade da espécie, com os fatores abióticos relacionados aos ecossistemas aquáticos e com a extensão da vegetação ripária dos trechos estudados.

\section{MATERIAL E MÉTODOS}

As amostragens foram conduzidas em três riachos tributários do rio Jacarezinho, bacia do rio das Cinzas, Alto rio Paraná. Em cada um dos riachos foram amostrados, trimestralmente, três pontos, com extensão de $50 \mathrm{~m}$ cada: riacho Água

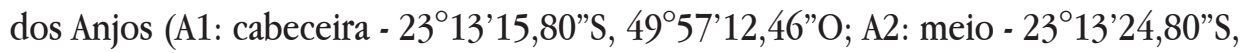

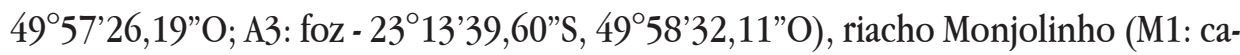

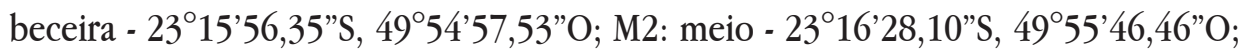

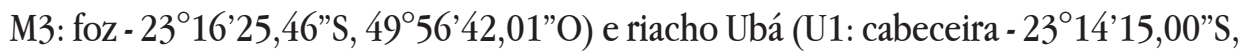

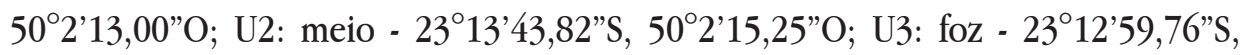
$50^{\circ} 2 \nmid 8,85^{\prime}$ '), no período de outubro de 2012 a julho de 2013 , totalizando quatro fases de campo (Figura 1). 


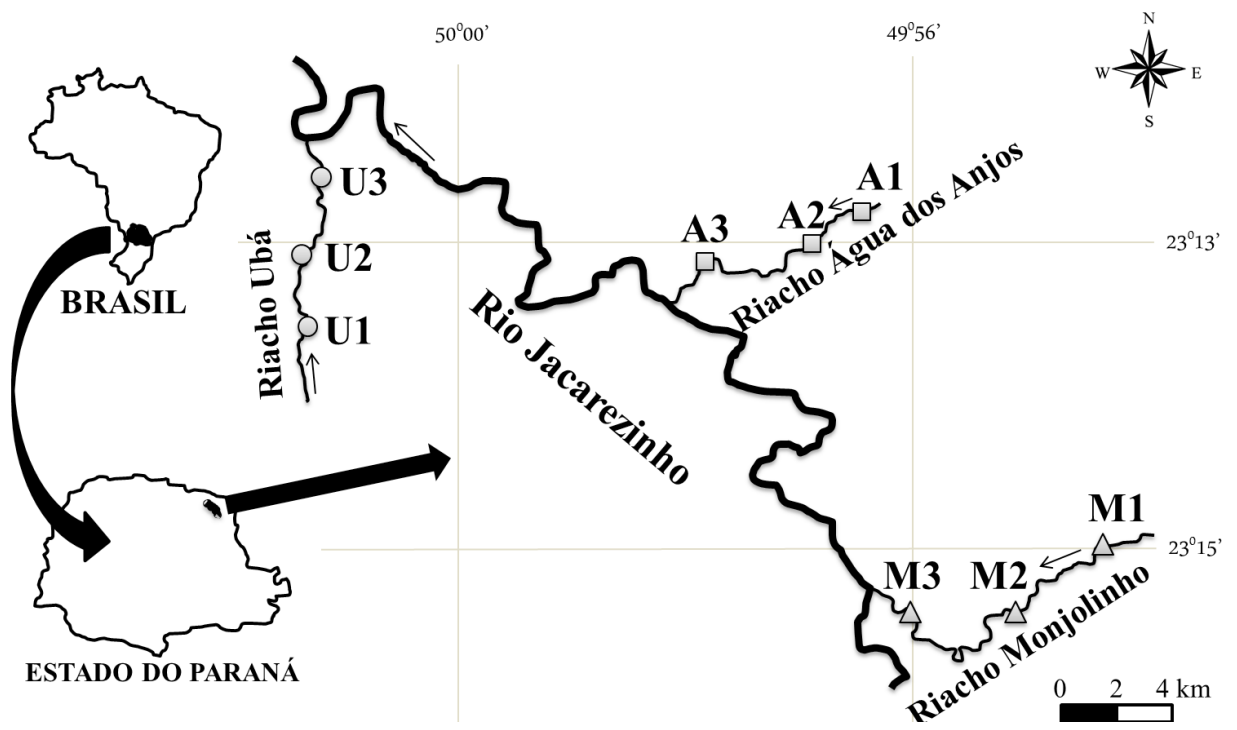

Figura 1. Unidades amostrais para coleta de peixes em riachos tributários do rio Jacarezinho, bacia do rio das Cinzas, sistema do Alto rio Paraná: riachos Ubá, Água dos Anjos e Monjolinho. As setas indicam a direção do fluxo da água.

As características físicas e hidrológicas aferidas em cada unidade amostral foram: largura e profundidade máximas do canal, com auxílio de uma trena de 10 metros, velocidade da água pelo método flutuador, vazão (PALHARES et al., 2007) e temperatura (Tecnopon ${ }^{\circledR}$ MPA-210P). Para os parâmetros químicos foram analisados: condutividade (Instrutherm ${ }^{\circledR}$ CD-860), oxigênio dissolvido (Politerm $®$ POL60) e pH (Tecnopon @ MPA-210P). Também foi verificada, por análise via satélite, a extensão aproximada da vegetação ripária em cada trecho estudado.

Foram analisados 2.601 indivíduos da espécie Bryconamericus iberingii, sendo 1.294 do riacho Água dos Anjos, 1.117 do riacho Monjolinho e 190 do riacho Ubá, capturados por meio de pesca elétrica com corrente alternada de 127 volts e 6 amperes (licença do Instituto Chico Mendes de Conservação da Biodiversidade, IBAMA - número: 30357-3). Foram realizadas a quantificação e a análise biométrica, sendo mensurados: biomassa total (em gramas), com auxílio de balança analítica, e comprimento padrão (em milímetros), com auxílio de paquímetro digital (Jomarca (B). Um lote da espécie coletada encontra-se depositado na coleção de peixes do Museu de Ictiologia do Núcleo de Pesquisas em Limnologia, Ictiologia e Aquicultura, da Universidade Estadual de Maringá (NUPELIA/UEM), sob o código "NUP14787". 
Referente à análise dos dados, primeiramente foi verificada a relação peso-comprimento, através da equação $\mathrm{y}=\mathrm{ax}$, onde: $\mathrm{y}=$ peso total $(\mathrm{g}), \mathrm{x}=\mathrm{com}$ primento padrão $(\mathrm{mm}), \mathrm{a}=$ constante de regressão relacionada com o grau de engorda do peixe e $b=$ coeficiente angular relacionado com o tipo de crescimento da população. Sendo assim: valores de $b=3$ indicam crescimento isométrico, com crescimento em comprimento equivalente ao ganho em peso; b $<3$ são considerados característicos de populações com crescimento alométrico negativo, com maior acréscimo em comprimento; e b $>3$, alométrico positivo, que significa um ganho maior em peso (HUXIEY; TEISSIER, 1936; ROCHA et al., 2003; RÊGO et al., 2008). Também foi estipulado o Fator de Condição Relativo (Kn) dos indivíduos coletados, utilizando os valores de peso e comprimento padrão (Ls) (WOOTTON; EVANS; MILLS, 1978). A fórmula de Kn é a seguinte: Wt/We, onde Wt = peso empiricamente registrado e We o peso teoricamente esperado para um dado comprimento. Os valores dos coeficientes de regressão a e b, utilizados nas estimativas dos valores esperados de peso $(\mathrm{We})$ pela equação We $=\mathrm{a}$.Ls $\mathrm{b}$, foram estimados através do ajustamento da curva da relação Wt/Ls (LE CREN, 1951).

Adicionalmente, foi verificado se ocorreram diferenças significativas quanto aos parâmetros de peso, comprimento padrão e Kn entre os riachos, utilizando Análises de Variância (ANOVA unifatorial). Por fim, análises de Spearman foram empregadas para correlacionar dados dos fatores abióticos, da extensão da vegetação ripária, das densidades de indivíduos (em cada trecho com 50 metros) e de Kn de $B$. iberingii nos riachos.

\section{RESULTADO E DISCUSSÃO}

A figura 2 mostra a relação peso-comprimento de $B$. iheringii nos três riachos estudados durante todo o período amostral. No riacho Monjolinho a correlação entre as duas variáveis supracitadas foi maior, com coeficiente de correlação de $\mathrm{r}=0,94$, do que nos riachos Água dos Anjos e Ubá, que apresentaram ambos, coeficiente de $\mathrm{r}=0,87$. O crescimento dos peixes nos riachos Água dos Anjos e Monjolinho pode ser considerado alométrico negativo (b $<3$ ), e no riacho Ubá, alométrico positivo $(\mathrm{b}>3)$. 

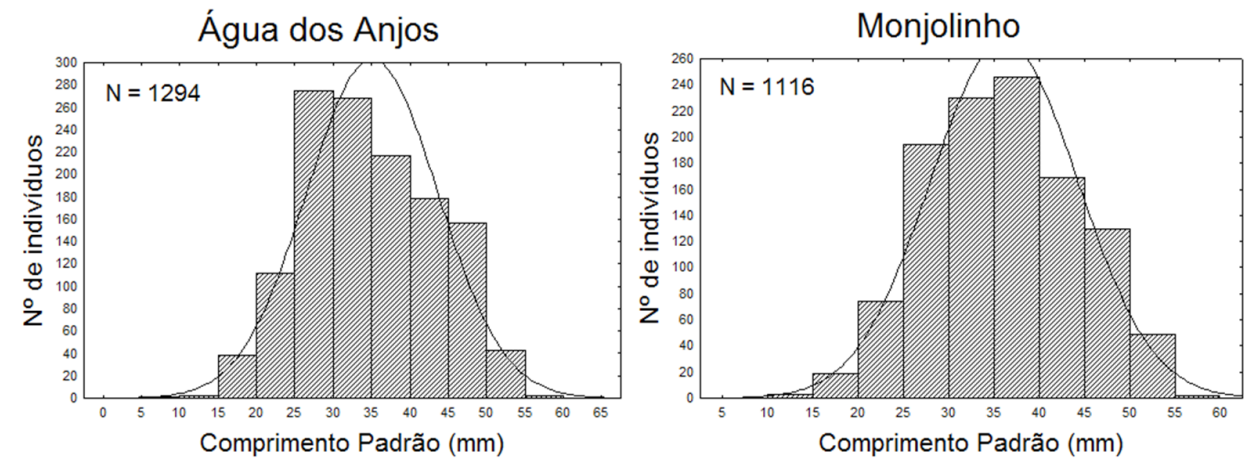

Ubá

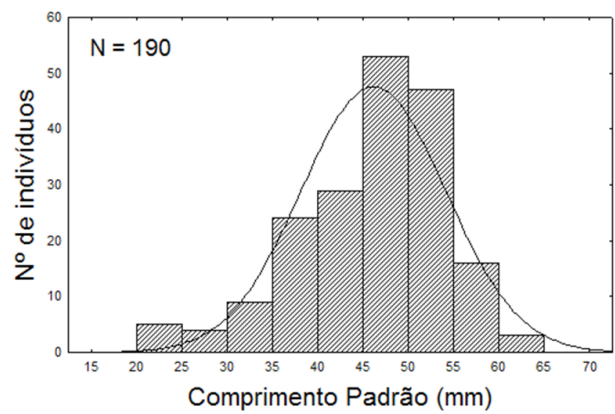

Figura 2. Relação peso-comprimento de peixes da espécie B. iberingii nos riachos Água dos Anjos, Monjolinho e Ubá, bacia do rio das Cinzas, Alto rio Paraná

Os espécimes de $B$. iheringii coletados no riacho Ubá foram significativamente mais pesados que os dos riachos Água dos Anjos e Monjolinho (Figura 3). Os indivíduos apresentaram um comprimento padrão variando de $6,43 \mathrm{~mm}$ a 56,61 $\mathrm{mm}$ (média $=34,90$ ) no riacho Água dos Anjos, de 14,04 mm a 55,57 mm (média $=35,97 \mathrm{~mm}$ ) no riacho Monjolinho, e de 21,22 $\mathrm{mm}$ a 62,06 (média $=45,98 \mathrm{~mm}$ ) no riacho Ubá (Figura 4). 

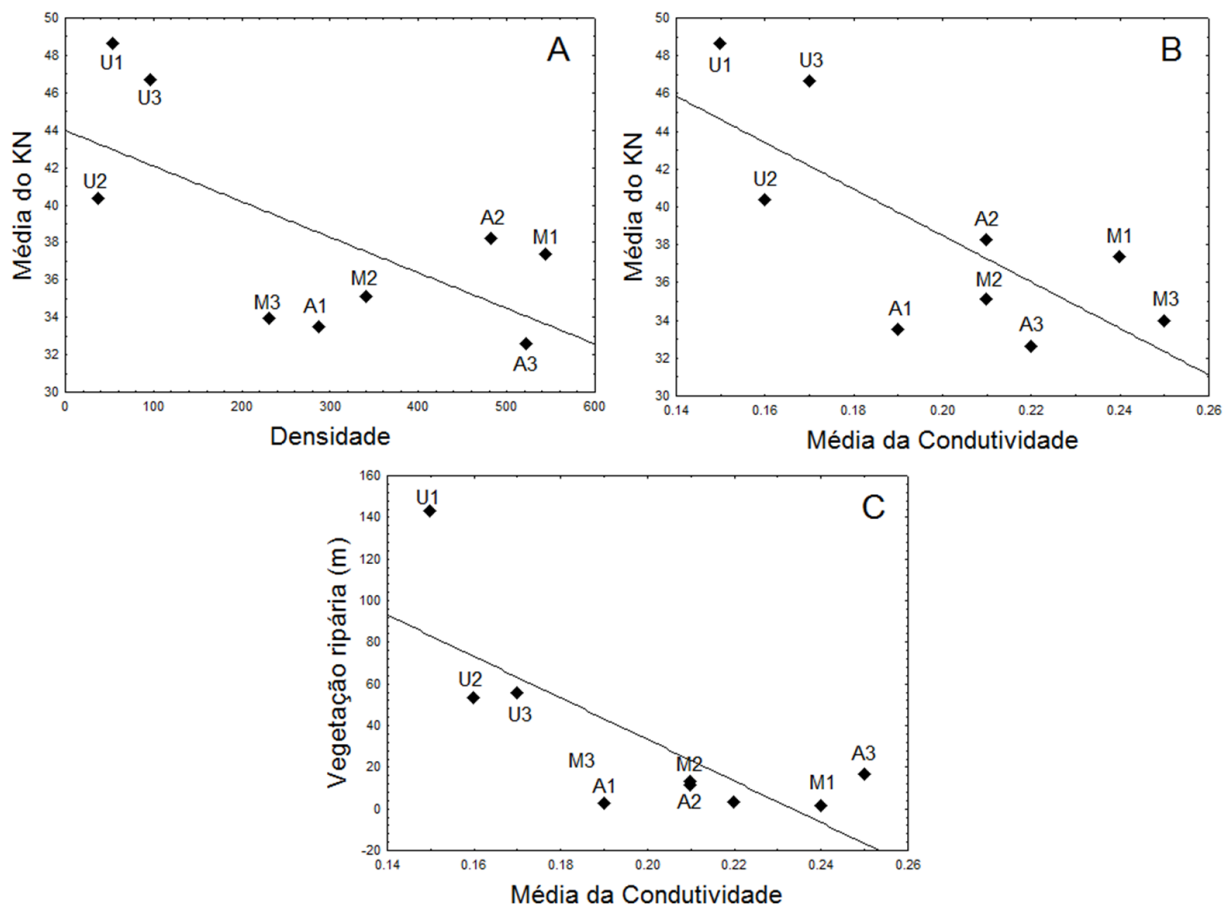

Figura 3. Diferenças (ANOVA Unifatorial) entre os valores do peso de indivíduos da espécie $B$. iheringii dos riachos Água dos Anjos, Monjolinho e Ubá, bacia do rio das Cinzas, Alto rio Paraná 

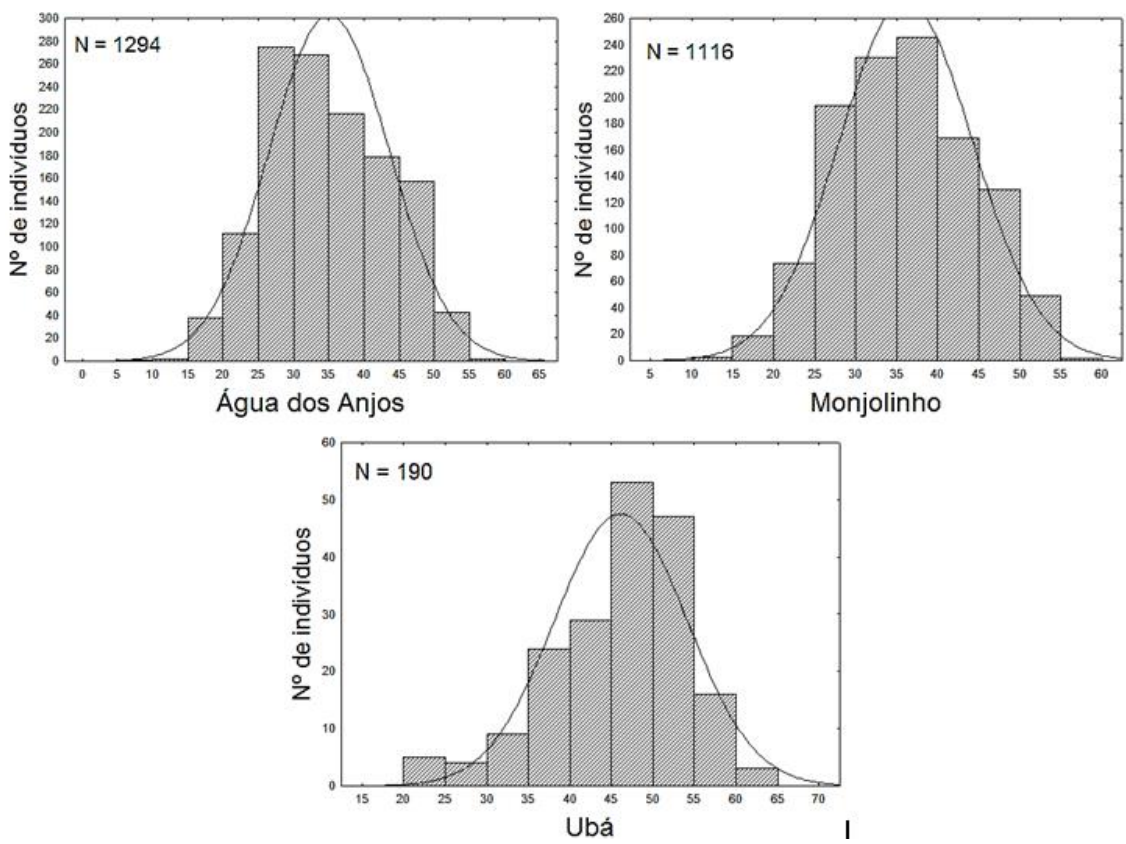

Figura 4. Distribuição de frequência de classes de tamanho em indivíduos da espécie $B$. iheringii dos riachos Água dos Anjos, Monjolinho e Ubá, bacia do rio das Cinzas, Alto rio Paraná.

Com relação ao Kn, foi encontrada diferença significativa $(p<0,05)$ entre os riachos, sendo que nos trechos do riacho Ubá (U1, U2 e U3) foram verificados maiores valores do que nos riachos Água dos Anjos e Monjolinho (Figura 5). Pelo índice de Spearman foi constatada uma correlação negativa entre o Kn e a densidade $(\mathrm{r}=0,62, p=0,05)$, o Kn e a condutividade $(\mathrm{r}=-0,68, p=0,04)$ e a condutividade e extensão da vegetação ripária ( $r=-0,79, p=0,01$; Figura 6 ). Não foram encontradas correlações significativas do Kn com as demais variáveis abióticas (largura, profundidade, velocidade da água, temperatura, oxigênio dissolvido e $\mathrm{pH}$ ). 


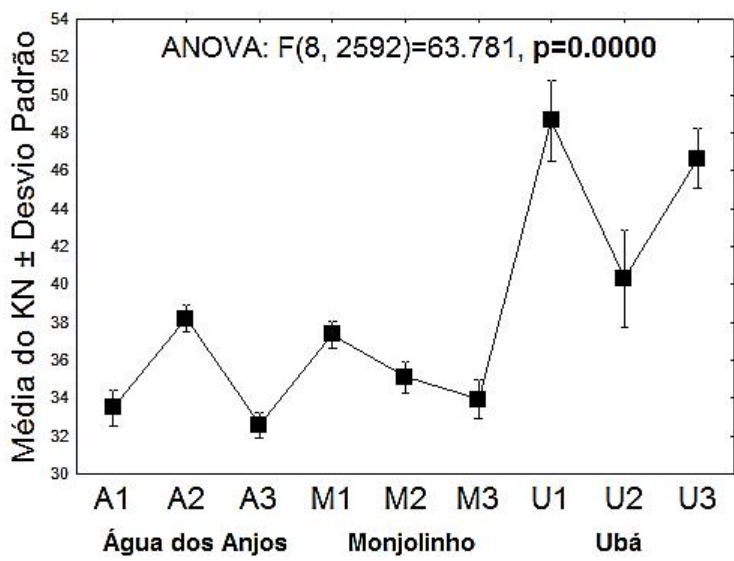

Figura 5. Diferenças nos valores de Kn (ANOVA Unifatorial) de peixes da espécie B. iheringii entre os riachos Água dos Anjos, Monjolinho e Ubá, bacia do rio das Cinzas, Alto rio Paraná.
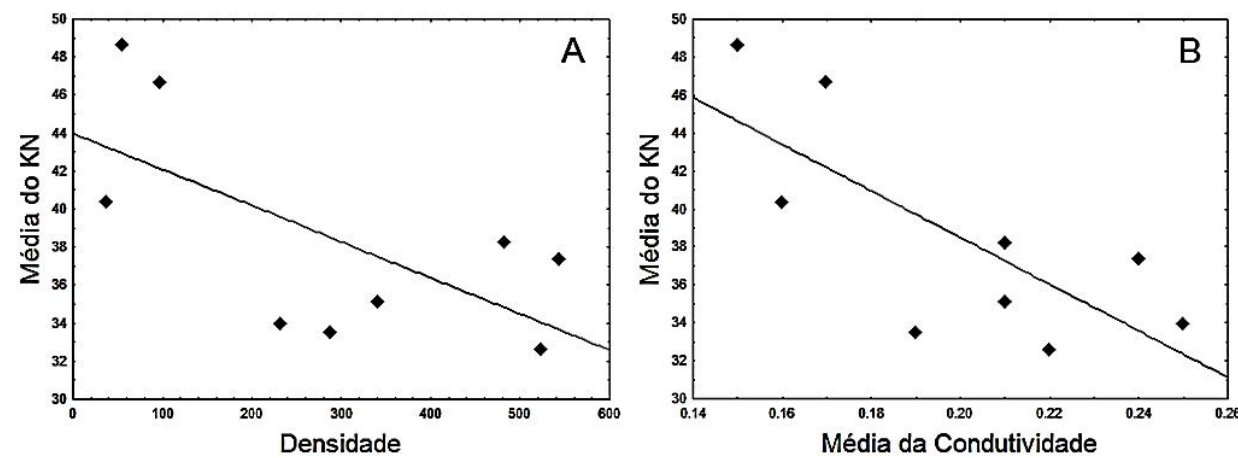

Figura 6. Correlação (Spearman) dos valores de Kn de peixes da espécie B. iheringii com a densidade (A) e a condutividade (B), e da condutividade com a extensão da vegetação ripária (C) em riachos da bacia do rio das Cinzas, Alto rio Paraná.

O crescimento alométrico negativo verificado nos peixes dos riachos Água dos Anjos e Monjolinho significa que ocorreu um acréscimo maior no comprimento do que no peso corporal dos indivíduos capturados nesses ambientes, e o crescimento alométrico positivo no riacho Ubá indica o contrário, ou seja, um maior ganho em peso do que em comprimento.

Também foi verificado um crescimento alométrico positivo em peixes da espécie $B$. iheringii capturados em córregos da bacia do rio Samborombón, província de São Vicente, Buenos Aires, Argentina (FERRIZ et al., 2010). 
Bryconamericus iheringii pode ser considerada uma espécie de hábito alimentar generalista, desta forma, as diferenças nas características ambientais do riacho Ubá, como a maior extensão de mata ripária e a ausência de assoreamento, podem ter sido importantes fatores para que os indivíduos conseguissem obter um maior acréscimo em peso do que em comprimento, pois esse riacho apresenta, supostamente devido à sua preservação, uma maior disponibilidade de recursos alimentares, como sementes e insetos terrestres na superfície, algas filamentosas no meio do corpo d'água, e detritos e outros invertebrados no fundo. Em ambientes com pouca ou nenhuma vegetação ripária, $B$. iheringii costuma se alimentar de detritos e quironomídeos, ingerindo junto sedimento resultante do assoreamento (CASATTI; LANGEANI; CASTRO, 2001; ORICOLLI; BENNEMANN, 2006). As variáveis abióticas determinam os recursos alimentares presentes no ambiente, que irão influenciar o estado nutricional, e consequentemente o $\mathrm{Kn}$, da população (BAGARINÃO; THAYAPARAN, 1986).

Foi possível verificar claramente que no riacho Ubá os indivíduos da espécie $B$. iheringii encontram-se mais pesados e compridos, o que ajudou a proporcionar também maiores valores de $\mathrm{Kn}$. Esse resultado foi influenciado principalmente pela condutividade, sendo que a menor condutividade no riacho Ubá proporcionou maiores Kn's dos peixes analisados. As florestas ripárias podem impedir a entrada de sedimentos (como os oriundos do assoreamento) e de pesticidas nos riachos, que escoam subterrânea e superficialmente, sendo assim, a falta de cobertura vegetal nas margens pode causar erosões e assoreamento, e consequente aumento da condutividade (CASATTI, 2010). Nesse sentido, pode-se dizer que a presença de vegetação ripária está causando uma diminuição da condutividade no riacho Ubá e, de forma independente, aumento de Kn dos peixes, através do favorecimento das trocas de material orgânico (folhas, troncos, frutos, sementes e insetos terrestres) entre o ambiente terrestre e aquático, influenciando positivamente sobre a alimentação e a capacidade de forrageio dos peixes, interferindo assim, em seu peso e bem-estar (PUSEY; ARTHINGTON, 2003; NAIMAN; DÉCAMPS; McCLAIN, 2005; BORDIGNON et al., 2015).

Nesse sentido, a condutividade, que mede a concentração de íons na água, pode indicar má qualidade ambiental, caracterizando-se como resultado do despejo 
de resíduos orgânicos ou então do assoreamento das margens, que ocorre devido à falta de vegetação ripária (FELIPE; SÚAREZ, 2010), afetando de forma significativa o crescimento dos organismos.

Outro fator que influenciou o Kn de $B$. iheringii entre os três riachos foi a densidade populacional, visto que em trechos com maiores densidades a média de Kn foi menor. $\mathrm{O}$ acréscimo na densidade populacional também pode estar relacionado com a perda da qualidade ambiental, pois as maiores densidades ocorreram justamente nos riachos menos preservados (sem ou com pouca mata ripária). De acordo com Caetano, Oliveira e Zawadzki (2016), B. iberingii é uma espécie normalmente mais abundante/densa em ambientes com baixa extensão de mata ripária (menor que $6 \mathrm{~m}$ ) e substrato instável, como areia, podendo até ser utilizada, cautelosamente, como indicadora desse tipo de ambiente. Sendo assim, a má qualidade ambiental pode provocar o aumento da densidade de $B$. iheringii, que por sua vez condiciona aumento na competição intraespecífica, principalmente por recursos alimentares, levando a uma diminuição na biomassa entre os indivíduos e, consequentemente, no Kn dos mesmos (RICKLEFS, 2010).

\section{CONSIDERAÇÕES FINAIS}

Portanto, a preservação da vegetação ripária em riachos revelou-se importante, aparentemente, na determinação de sua condutividade, e afetou os parâmetros ecológicos da espécie $B$. iberingii tanto com relação ao quociente peso-comprimento quanto ao Kn. Sendo assim, alterações estruturais em corpos d'água podem afetar negativamente o equilíbrio das populações e consequentemente de todo o ecossistema. As análises de populações de peixes relacionadas ao peso-comprimento e ao Kn podem ser bons parâmetros complementares para entender a resposta dos organismos às alterações ambientais em riachos.

\section{AGRADECIMENTOS}

Os autores agradecem ao Grupo de Estudos em Recursos Hídricos e Ecologia Aplicada (GEPRHEA) da Universidade Estadual do Norte do Paraná, pelo suporte 
técnico e logístico, ao Núcleo de Pesquisas em Limnologia, Ictiologia e Aquicultura (Nupélia) pela confirmação na identificação da espécie de peixe coletada; à Coordenação de Aperfeiçoamento de Pessoal de Nível Superior (CAPES) e ao Programa de Pós-Graduação em Biologia Evolutiva da Universidade Estadual do Centro-Oeste, pelo suporte financeiro e pela bolsa de mestrado concedida ao primeiro autor.

\section{REFERÊNCIAS}

AGBOOLA, J. I.; ANETEKHAI, M. A. Length-weight relationships of some fresh and brackish water fishes in Badagry Creek, Nigeria. Journal of Application Ichthyology, v. 24, p. 623-625, 2008.

ALEKSEEVSKII, N. I.; GRINEVSKII, S. O.; EFREMOV, P. V.; ZALAVSKAYA, M. B.; GRIGOR'EVA, I. L. Small Rivers and the ecological State of an Area. Water Research, v. 30, n. 5, p. 540-549, 2003.

ANDRADE-TALMELLI, E. F.; FENERICH-VERANI, N.; VERANI, J. R. Fator de condição relativo $(\mathrm{kn})$ : um critério para selecionar Fêmeas de piabanha, Brycon insignis (steindachner, 1876) (pisces: Bryconinae), para indução reprodutiva. Boletim do Instituto de Pesca, v. 25, p. 95-99, 1999.

BAGARINÃO, T.; THAYAPARAN, K. The length-weight relationship, food habitats and condition factor of wild juvenile milkfish in Sri Lanka. Aquaculture, v. 55, p. 241246, 1986.

BORDIGNON, C. R.; CASATTI, L.; PÉREZ-MAYORGA, M. A.; TERESA, F. B.; BREJÃO, G. L. Fish complementarity is associated to forests in Amazonian streams. Neotropical Ichthyology, v. 13, n. 3, p. 579-590, 2015.

CAETANO, D. L. F.; OLIVEIRA, E. F.; ZAWADZKI, C. H. Fish species indicators of environmental quality of Neotropical streams in southern Brazil, upper Paraná river basin. Acta Ichthyologica Et Piscatoria, v. 46, n. 2, p. 87-96, 2016.

CASATTI, L. Alterações no Código Florestal Brasileiro: impactos potenciais sobre a ictiofauna. Biota Neotropica, v. 10, n. 4, p. 31-34, 2010. 
CASATTI, L.; LANGEANI, F.; CASTRO, R. M. C. Peixes de riacho do Parque Estadual Morro do Diabo, bacia do alto rio Paraná, SP. Biota Neotropica, v. 1, n. 1/2, p. 1-15, 2001.

CIFUENTES, R.; GONZÁLEZ, J.; MONTOYA, G.; JARA, A.; ORTÍZ, N.; PIEDRA, P.; HABIT, E. Relación longitud-peso y factor de condición de los peces nativos del río San Pedro (cuenca del río Valdivia, Chile). Gayana Especial, p. 101-110, 2012.

FELIPE, T. R. A.; SÚAREZ, Y. R. Influência dos fatores ambientais nas comunidades de peixes de riachos em duas microbacias urbanas, Alto Rio Paraná. Biota Neotropica, v. 10, n. 2, p. 143-151, 2010.

FERRIZ, R. A.; BENTOS, C. A.; LÓPEZ, G. R.; FERNÁNDEZ, E. M. Algunos aspectos biológicos de Bryconamericus iberingii (Ostariophysi: Characidae) en dos arroyos de la alta cuenca del río Samborombón, Argentina. Revista del Museo Argentino de Ciencias Naturales, v. 12, n. 2, p. 109-116, 2010.

GONÇALVES, C. S.; BRAGA, F. M. S. Changes in ichthyofauna composition along a gradient from clearwaters to blackwaters in coastal streams of Atlantic forest (southeastern Brazil) in relation to environmental variables. Neotropical Ichthyology, v. 10, n. 3, p. $675-684,2012$

GRAÇA, W. J.; PAVANELLI, C. S. Peixes da planície de inundação do alto rio Paraná e áreas adjacentes. Maringá: Eduem, 2007.

GUIDELLI, G.; TAVECHIO, W. L.; TAKEMOTO, R. M.; PAVANELLI, G. C. Relative condition factor and parasitism in anostomid fishes from the floodplain of the Upper Paraná River, Brazil. Veterinary Parasitology, v. 177, n. 1-2, p. 145-151, 2011.

HAHN, N. S.; FUGI, R.; ADRIAN, F. Trophic ecology of the fish assemblages. In: THOMAZ, S. M.; AGOSTINHO, A. A.; HAHN, N. S. (Eds). The upper Paraná River and its Floodplain: physical aspects, ecology and conservation. Leiden, The Netherlands, Backhuys Publishers, p. 247-269, 2004.

HAUER, F. R.; LAMBERTI, G. A. Methods in stream ecology. San Diego: Academic Press, 1996. 
HUXLEY, J. S.; TEISSIER, G. Terminology of relative growth. Nature, v. 137, p. 780$781,1936$.

LE CREN, E. D. The length-weight relationship and seasonal cycle in the gonad weight and condition in the perch (Perca fluviatilis). Journal of Animal Ecology, v. 20 , p. 201-219, 1951.

NAIMAN, R. J.; DÉCAMPS, H.; McCLAIN, M. E. Riparia: ecology, conservation and management of streamside communities. Elsevier Academic Press, Burlington, 2005.

ORICOLLI, M. C. G.; BENNEMANN, T. Dieta de Bryconamericus iheringii (Ostariophysi: Characidae) em riachos da bacia do rio Tibagi, Estado do Paraná. Acta Scientiarum. Biological Sciences, v. 28, n. 1, p. 59-63, 2006.

PALHARES, J. C. P.; RAMOS, C.; KLEIN, J. B.; LIMA, J. M. M.; MULLER, S.; CESTONARO, T. Medição da Vazão em Rios pelo Método do Flutuador. Comunicado técnico Embrapa, p. 1-4, 2007.

PUSEY, B. J.; ARTHINGTON, A. H. Importance of the riparian zone to the conservation and management of freshwater fish: a review. Marine and Freshwater Research, v. 54, p. 1-16, 2003.

RÊGO, C. L.; PINESE, O. P.; MAGALHÃES, P. A.; PINESE, J. F. Relação peso-comprimento para Prochilodus lineatus (Valenciennes, 1836) e Leporinus friderici (Bloch, 1794) (Characiformes) no reservatório de Nova Ponte-EPDA de Galheiro, rio Araguari, MG. Revista Brasileira de Zoociências, v. 10, n. 1, p. 13-21, 2008.

RIBEIRO, M. D.; TERESA, F. B.; CASATTI, L. Use of functional traits to assess changes in stream fish assemblages across a habitat gradient. Neotropical Ichthyology, v. 14, n. 1, e140185, 2016.

RICKLEFS, R. E. Competição. In: RICKLEFS, R. E. Economia da Natureza. $\sigma^{\mathrm{a}}$ ed. Rio de Janeiro: Guanabara Koogan, p. 291-306, 2010.

ROCHA, M. A.; RIBEIRO, E. D. A.; MIZUBUTI, I. Y.; DA SILVA, L. D. F.; BIGNARDI, A. B.; DOMINGUES, A. R. Relações entre as características de crescimento na tilápia (Oreochromis nilotica). Semina: Ciências Agrárias, v. 24, n. 1, p. 119-122, 2003. 
ROSA, R. R.; CAETANO, D. L. F.; BELLAY, S.; DE MORAES, V. R.; VIEIRA, F. E. G. Diversidade de peixes de tributários do reservatório de Chavantes, PR, bacia do alto rio Paraná. Biotemas, v. 29, n. 2, p. 33-43, 2016.

SCHNEIDER, J. C.; LAARMAN, P. W.; GOWING, H. Length-weight relationships. In: SCHNEIDER, J. C. (Ed.). Manual of fisheries survey methods II: with periodic updates. Michigan Department of Natural Resources, Fisheries Special Report 25, Ann Arbor, p. 1-16, 2000.

SHIBATTA, O. A.; ORSI, M. L.; BENNEMANN, S. T.; SILVA-SOUZA, A. T. Diversidade e distribuição de peixes na bacia do rio Tibagi. In: MEDRI, M. E.; BIANCHINI, E.; SHIBATTA, O. A.; PIMENTA, J. A. (Eds.). A bacia do rio Tibagi. Londrina: UEL, p. 403-423, 2002.

SÚAREZ, Y. R.; SOUZA, M. M.; FERREIRA, F. S.; PEREIRA, M. J.; SILVA, E. A.; XIMENES, L. Q. L.; AZEVEDO, L. G.; MARTINS, O. M.; LIMA-JUNIOR, S. E. Patterns of species richness and composition of ish assemblages in stream of the Ivinhema River basin, Upper Paraná River. Acta Limnologica Brasiliensia, v. 23, n. 2, p. 177 188, 2011.

TREER, T.; SPREM, N.; TORCU-KOC, H.; SUN, Y.; PIRIA, M. Length-weight relationships of freshwater fishes of Croatia. Journal of Application Ichthyology, $\mathrm{v}$. 24, p. 626-628, 2008.

UIEDA, V. S.; CASTRO, R. M. C. Coleta e fixação de peixes de riachos. In: CARAMASCHI, E. P.; MAZZONI, R.; PERES-NETO, P. R. (Eds.). Ecologia de Peixes de Riachos. Oecologia Brasiliensis, v. 6, p. 1-22, 1999.

VANNOTE, R. L.; MINSHALL, G. W.; CUMMINS, K. W.; SEDELL, J. R.; CUSHING, C. E. The river continuum concept. Canadian Journal of Fisheries and Aquatic Sciences, v. 37, p. 130-137, 1980.

WOOTTON, R. J.; EVANS, G. W.; MILLS, L. Annual cycle in female three-spined sticklebacks (Gasterosteus aculeatus) from an upland and lowland population. Journal of Fish Biology, v. 12, p. 331-343, 1978. 
ZENI, J. O.; CASATTI, L. The influence of habitat homogenization on the trophic structure of fish fauna in tropical streams. Hydrobiologia, v. 726, p. 259-270, 2014.

Recebido em: 23/12/2015 Aceito em: 27/10/2017 